\title{
Millimetre Wave Reflectarray Antenna Unit Cell Measurements
}

\author{
G. Ahmad*, T. W. C. Brown*, C. I. Underwood*, T. H. $\operatorname{Loh}^{\dagger}$ \\ * University of Surrey, UK, g.ahmad@ surrey.ac.uk, ${ }^{\dagger}$ National Physical Laboratory (NPL) ,UK
}

Keywords: mm-waves, reflectarrays, unit cell, measurement.

\begin{abstract}
Reflectarray antennas are a potential candidate solution to realize high gains at millimetre waves (mm-waves). A reflectarray contains a large number of spatially illuminated unit cells. The performance of a good reflectarray design is manifested by the behaviour of its comprising unit cells. An established technique to characterise a unit cell is by placing it inside a waveguide to achieve periodic boundary conditions. This usually requires custom waveguide products; making the tests difficult and expensive. Additionally, when the unit cells are reconfigurable as in a smart reflectarray it is hard to take the DC bias lines out of the waveguide without using custom made waveguide parts. This contribution address the issue of unit cell placement inside the waveguide and proposes simple unit cell structures to avoid custom made waveguide parts. The idea was verified by measuring a series of unit cells at mm-waves in various configurations and a practically acceptable agreement was found. The proposed structures greatly simplify the reconfigurable unit cell testing.
\end{abstract}

\section{Introduction}

At millimeter waves ( $\mathrm{mm}$ waves) high antenna gain is the primary determinant of a communication link operation. Parabolic reflectors and phased arrays are the historically proven solutions to achieve high gain. More recently, the reflectarrays by combining the best features of both historic technologies are becoming a potential candidate solution for future communication systems. Reflectarrays are planar in nature and benefit from spatial illumination of its unit cells to provide compactness and reduced system losses. A smart reflectarray facilitates the integration of reconfiguration techniques in its unit cells at antenna radio frequency (RF) plane to further reduce the losses associated with such a reconfiguration. In superb designs of passive as well as active/ smart reflectarrays, the overall performance is dominated by unit cells' characteristics.

Therefore, significant attention is focused on realizing unit cells that can achieve the required performance characteristics. To gain confidence in reflectarray fabrication facility, it is necessary to characterize the fabricated unit cells against sensibly performed electromagnetic (EM) simulations. The tolerances then can be identified and accommodated in the design to be manufactured to produce the desired end results. This necessitates the measurements of manufactured unit cells. The well-known waveguide test setup needs customization to accommodate unit cell(s) to carry out measurements. Similarly, an increasing trend of smart reflectarrays for simultaneous realization of high gains and wide angle beam scans further necessitates the characterization of reconfigurable unit cells. To provide DC biasing for control devices in a reconfigurable unit cell inside a waveguide present potential challenge and again customized waveguide parts are required. The customization of waveguide structure used in measurements is subject to the unit cell design. Commonly used methods implement trenches [1] in the waveguide short plate to hold unit cell(s) in place during measurements. Similarly holes or trenches are made in waveguide or short plate [2-6] respectively to route DC bias lines to the unit cell. It is a necessary however, cumbersome process.

It was felt necessary to modify the unit cell supporting structure such that commercial off the shelf (COTS) waveguide setup can be used for any type of unit cells at a certain frequency band. This simplifies the test waveguide fixture requirements and avoids customization. The issue of proper orientation of unit cell(s) during measurements gets resolved. Additionally, same waveguide structure can be used for passive and active unit cell measurements. For such an implementation one need to understand importance of measured quantities in a unit cell test. A reflectarray unit cell is mainly characterized by its reflection response. Unit cell losses contribute to reflectarray loss. However, the loss in reflectarray can be approximated by an average of the losses in its unit cells. In a well-designed unit cell in the over coupled region of operation [7], the reflection loss is less than few $\mathrm{dBs}$ and in most cases is deterministic in terms of substrate parameters. As the parabolic behavior is emulated by control of reflection phases from locations of unit cells in a reflectarray; therefore reflection phase response is the key parameter to be characterization in a unit cell measurement campaign.

We have presented a simplified and unified solution to circumvent the customization problem encountered in unit cell performance characterization using waveguide test setup. In section 2, the basic operation of a reflectarray and desirable unit cell properties are described. Commonly used structures for unit cell measurement are the subject of section 3. Section 4 presents the proposed structures. Unit cell measurements are presented in section 5. Measured results using proposed structures are compared with the commonly used measurement technique in this section. Finally, section 6 concludes this contribution.

\section{Reflectarray Operation \& Unit Cells}

A reflectarray in its basic form is comprised of a source (feed horn) antenna and a reflecting surface consisting of multiple unit cells. As shown in Fig. 1, the source antenna, with its phase center located at array focal point $(0,0, f)$, where $f$ is the 
focal length; spatially illuminates the reflectarray unit cells. Incoming rays from the source antenna are collimated by applying a phase correction to the scattered field at each unit cell. A constant phase of the entire scattered field is achieved in a plane normal to the direction of desired pencil beam as expressed by [8]:

$$
\frac{2 \pi}{\lambda_{0}}\left(r_{m n}-\vec{R}_{m n} \cdot \hat{r}_{b}\right)-\Delta \Phi_{m n}=2 \pi N
$$

where $\lambda_{0}$ is free space wavelength, $\vec{r}_{m n}$ is the position vector of $m n^{\text {th }}$ element/unit cell relative to $(0,0, f), \quad \vec{R}_{m n}$ is the position vector of $m n^{\text {th }}$ element relative to $(0,0,0), \hat{r}_{b}$ is the direction vector of the desired pencil beam, $N=1,2,3 \ldots$., and $\Delta \Phi_{m n}$ is the phase shift introduced by $m n^{\text {th }}$ unit cell of reflectarray to its scattered field relative to the incident field.

Due to scattered field's phase control at each unit cell, the reflectarrays can have versatile functionality, for instance a contoured beam or multiple pencil beams can be synthesized, and multiple feeding sources can be accommodated. The unit cell design, their layout in a reflectarray and phase control can be exploited to achieve good cross polarization levels. Most of the efforts in a reflectarray design are dedicated to realize unit cells to synthesize the desire phase characteristics as a result of variations in suitable parameter(s).

The unit cell properties mainly of interest are their reflection response (magnitude \& phase) and polarization purity. To achieve perfect reflection, the internal losses in a unit cell should be minimized to ensure the reflected field's magnitude is approximately equal to that of the incident field. However, the substrate loss tangent and conductor surface roughness of a printed circuit board (PCB) introduces significant loss, thereby lowering the value of reflection coefficient magnitude from its ideal value. In addition to these losses, the substrate thickness selection plays a major role in the unit cell's reflection loss.

To accommodate the geometry of a reflectarray, its unit cells should provide a large range of phases in excess of $360^{\circ}$. Reflection phase versus frequency response should be as linear as possible to achieve wider operational bandwidth. To avoid radiation pattern deterioration in large reflectarrays, the geometrical transitions should be small and continuous; it is particularly important in cases where reflection phase transitions to zero (a lower value) after the $360^{\circ}$ phase step. Reflection phase change in response to a corresponding change in the unit cell property (e.g. length or dielectric constant) should be low to accommodate the manufacturing tolerances and to avoid difficulties in phase control. In a practical reflectarray, all the unit cells are not illuminated by a normal incident field; the reflection phase from a unit cell should be a weak function of field incident angle.

Unit cell polarization purity should be high to meet the overall required cross polarization specification at reflectarray level. Cross polarization is addressed at both; unit cell and reflectarray levels. The orientation of unit cells in a reflectarray can be exploited in achieving lower levels of cross polar reflected fields [9].

Futuristic smart reflectarrays are expected to accommodate the reconfiguration of antenna properties e.g. frequency, polarization, beamwidth, bandwidth, or pencil beam scanning. In addition to the above mentioned characteristics of the unit

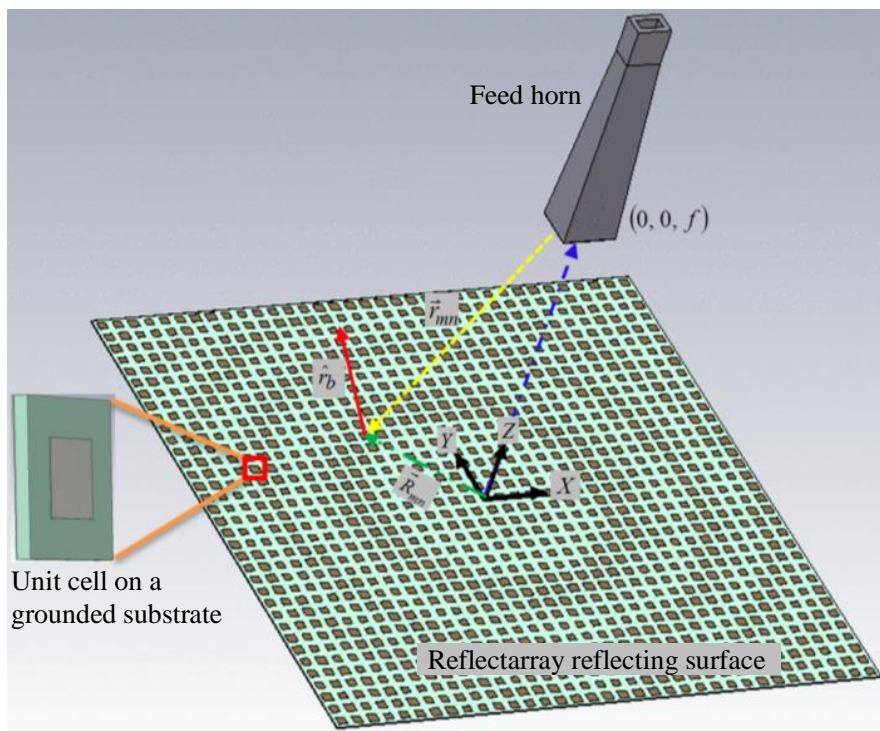

Fig. 1. A typical printed microstrip reflectarray on a grounded substrate

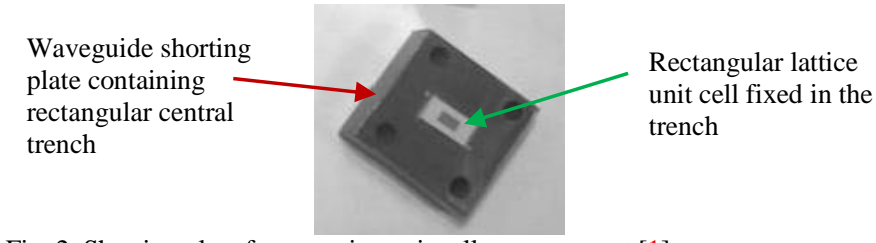

Fig. 2. Shorting plate for a passive unit cell measurement [1]

cell, its design should provide the sufficient flexibility to accommodate such a reconfiguration. An aspect particularly important to $\mathrm{mm}$-wave reflectarray design is the number of control elements required to achieve the desired reconfiguration property at unit cell level. As the unit cell physical size becomes comparable to the size of discrete electronic components, accommodation of multiple control elements becomes problematic and may result in array performance degradation due to increased inter element distance. Also the DC biasing of many control elements further complicate the design. Finally, the unit cell design should be simple enough to get manufactured at mm-waves frequencies without the severe performance degradation.

\section{Measurement Structures for Unit Cells}

This section provides an overview of the commonly used waveguide measurement structures for a unit cell. To measure passive unit cells it is a normal practice to make a trench in the shorting plate whose dimensions are comparable to that of the unit cell as shown in Fig. 2. A unit cell is placed inside the trench and is made to stick to the shorting plate using sticky glue etc. Proper orientation and positioning of a tiny millimeter unit cell may become problematic, which would lead to imperfect measured results. Additionally, shorting plates with a trench are custom products, which are not provided with the waveguide calibration kits. The shape of trench would limit the type of unit cells that can be measured and changing the shape of unit cell e.g. rectangular to square in this case would need a square trench shorting plate which is not a very elegant solution and would increase the cost.

The active unit cell measurement present a real challenge as the DC bias lines are required to be taken out of the waveguide structure. A typical such structure is shown in Fig. 3 where a 


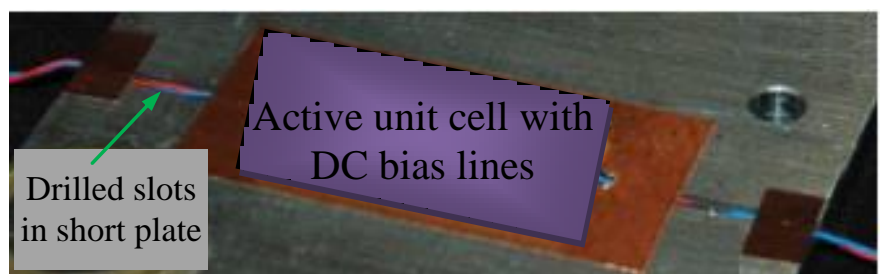

Fig. 3. Measurement setup of an active unit cell using slots in the waveguide short plate [2]

reconfigurable unit cell is mounted on the waveguide (WG) short plate and slots are provided for DC bias lines.

Similar structures can be observed in [3-6]. In a mm-wave real reflectarray environment where tiny physical features and constrained inter-element spacing become key concerns, the provision and routing of DC bias lines for thousands of unit cells on the same layer as that of unit cells present significant challenges and is very impractical as it leads to antenna performance degradation. It often leads to using via holes to transfer the DC bias lines to the other layers behind the radio frequency $(\mathrm{RF})$ ground plane of a unit cell where their routing is easy and more practical. These via holes remain unaddressed when using the structures discussed above. Taking the DC bias lines out the waveguide is mainly possible by cutting through hole(s) in the short plate.

In short the emulation of an active unit cell measurement setup that should resemble the actual environment in the reflectarray is a potentially difficult task particularly considering the DC bias lines. This issue is particularly important for $\mathrm{mm}$-waves where the geometrical features are tiny and there is not enough space to route DC lines on the same layer as that of the unit cell.

\section{Proposed Measurement Structures}

This section provides the details of proposed simplified and unified measurement structures applicable to mm-waves. The proposed structures are valid for reflection phase characterization of mm-wave unit cells. Fig. 4 displays the unit cells used in current study. We have explained our findings using square reflecting patch geometry to support dual polarization in our experimentation. The procedure is equally valid for a single polarized patch. In Fig. 4(a), a unit cell is shown with its outer boundaries. Outer boundaries help as guideline for slicing the fabricated unit cell to fit inside the test waveguide. Inner side dimensions of this boundary are equal to the wider dimension ' $a$ ' of the test waveguide. In our case we re-used rectangular to square waveguide transition (actually made for another experiment) to fit the square unit cell geometry. Therefore, both side dimensions were ' $a$ ' in this case. For single polarized cells, a common rectangular waveguide with ' $a$ ' $x$ ' $b$ ' can be used. Fig. 4 (b)-(c) displays unit cells fabricated on an enlarger lattice. These unit cells incorporate the waveguide adapter for mounting them to the test waveguide. Size of the unit cell can be adjusted to conform to type (circular/rectangular) and size of waveguide adapter according to the frequency of operation. At mm-waves alignment of waveguide parts need alignment dongle pins in addition to mounting screws. Smaller circular markings are holes for these pins while bigger markings are holes for mounting screws. After drilling these holes, the copper /

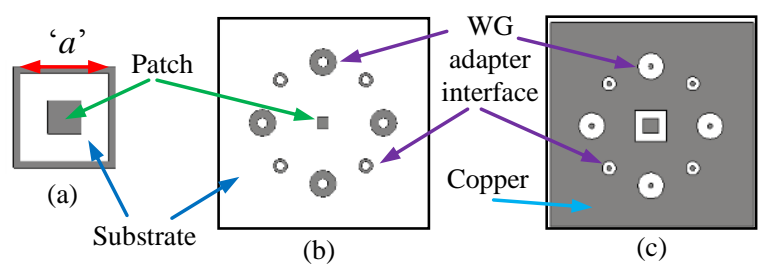

Fig. 4. Square reflecting patch unit cells on a grounded substrate, (a) square patch with outer boundary, inner side lengths of boundary are wider dimension ' $a$ ' of the waveguide, (b) unit cell with waveguide adapter interface; circular marking are holes for alignment dongle pins / mounting screws, (c) unit cell with waveguide adapter and copper on top surface.

substrate area as shown in Fig. 4 (b) and (c) respectively for adapter mounting interface disappears. In Fig. 4 (c) there is copper on top surface that makes an electrical contact with the waveguide metal flange surface. In all three cases substrate's rear side acts as metal ground plane. Unit cells shown in Fig. 4 (b)-(c) have the advantage to facilitate excellent alignment as compared to the one shown in Fig. 4 (a). In addition to solving the unit cell mounting problem in waveguide, the unit cell structures in Fig. 4 (b)-(c) provide the capability to take the DC biasing contacts out of the waveguide setup.

\section{Unit Cell Measurements}

A series of unit cells from Fig. 4 (a)-(c) were fabricated and measured as described in this section. To identify the effect of metal short plate, measurements were made in presence and absence of waveguide shorting plate. A unit cell of type Fig. 4 (a) sliced to the dimensions of square waveguide was placed at its end where copper on unit cell's rear side was in line with the waveguide adapter as shown in Fig. 5. The metal short plate was fixed and measurement was taken. In the same configuration metal plate was removed and measurement was repeated. There was hardly any significant difference observed in reflection coefficient's phase; the same is true for the reflection coefficient's magnitude. It indicates when the unit cell is made to fit inside the waveguide its own rear ground plane is enough for reflections and there is no need for the metal short plate behind it. Also it is worth noting here that in our experiments the substrate was RO5880 having 10 mil thickness and operating at $60 \mathrm{GHz}$. To relate this case with the commonly used trench short plate measurements where the unit cell reflecting patch is in-line with the waveguide flange, it was observed that the change in phase due to substrate thickness was negligible for practical purposes when it is placed inside or outside the waveguide. This was verified by repeated measurements by holding the unit cell in a way that the substrate mainly remains outside the waveguide. In this case the energy leakage from waveguide was not very significant and can be considered acceptable for practical purposes.

The measurement of unit cells types in Fig. 4 (b)-(c), is shown in Fig. 6. Here waveguide short plate is shown on the rear side which serves mainly as a support for substrate during the measurements. Any other support material e.g. acrylic would serve the purpose. The measured results for reflection coefficient's phase are compared in in Fig. 7 where a close agreement can be seen for all three cases. A comparable reflection loss was observed for all three cases around their 


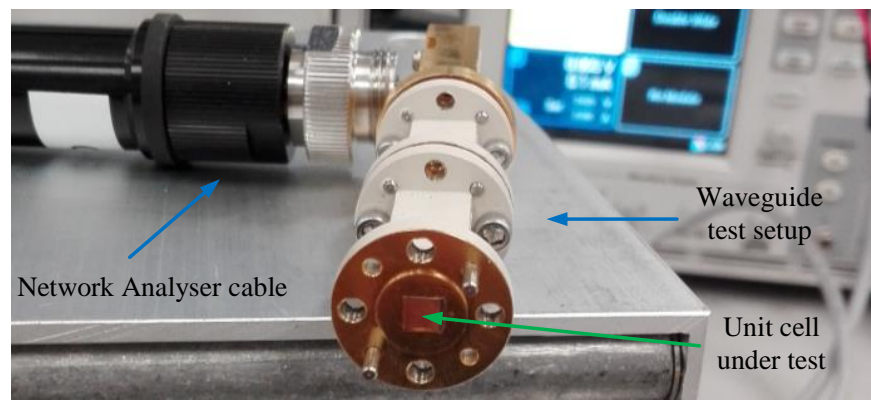

Fig. 5. A passive unit cell under test in waveguide test setup. The unit cell is sliced to make it fit in the square waveguide. Slicing had minor imperfections. Unit cell was measured twice; once using the metal short plate at the end and once as shown without metal short plate.
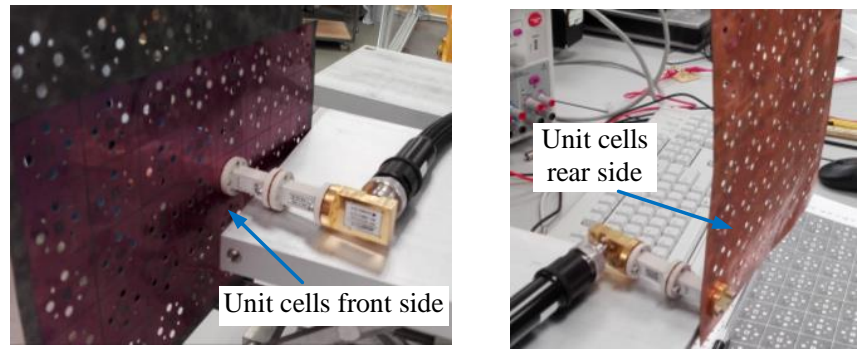

Fig. 6. Passive unit cells designs following Fig. 4(b)-(c) under test.

respective resonances. Table 1 provides a comparison of measured results of unit cell types shown in Fig. 4 (a)-(c). The unit cell of type Fig. 4 (a); whose measurement is shown in Fig. 5, was used as a reference case for comparison. A slight shift in the resonant frequency, hence the reflection phase was observed during measurement for unit cell types shown in Fig. 4 (b)-(c). In Table 1, frequency deviation indicates this shift in frequency w.r.t. the base case. Similarly, phase deviation indicates the difference of phase w.r.t. the base case measured at $59.23 \mathrm{GHz}$. One possible reason for these shifts is the printing accuracy of our photo lithography facility used in PCB fabrication which is in fact a low frequency facility. It was observed during facility characterization measurements that there are slight variations in phase due to location dependence printing of the unit cells. Another reason is the change in type of unit cells. However, for practical purposes these measurements considerably agree very well keeping in view these are $\mathrm{V}$ band measurements where slight imperfection can lead to greater errors.

These measurements indicate that reflection phase (which is of the main interest for reflectarray design) of unit cells can be very well characterized using the proposed structures. The proposed structures provide a unified solution for any type/shape of unit cells as only the reflecting patch itself gets changed and rest of the supporting structure remains the same. Additionally, unit cells can be placed at the desired location and orientation easily without the need of trenches etc. in the waveguide short plate.

These measurements greatly facilitate the measurement of active unit cells where provision of DC biasing inside the waveguide presents potential challenges. The proposed topology in Fig. 4 (c) was used in active unit cell designs at V band. The design of these active unit cells is beyond the scope of this contribution and is in patenting stage, therefore not disclosed here. However, the measurement topology for active unit cells at V band is discussed here.

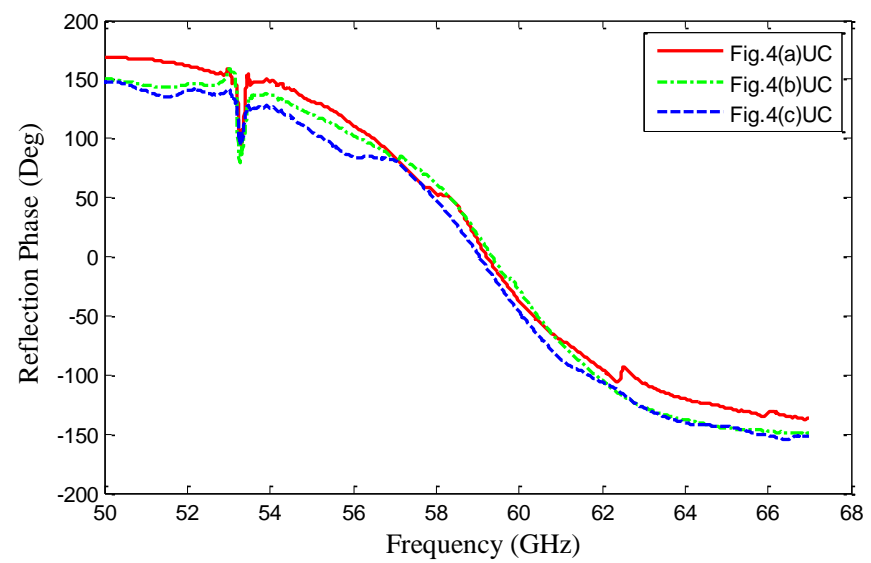

Fig. 7. Reflection phase measurements for unit cell (UC) types shown in Fig. 4 (a)-(c); a very good agreement can be observed considering these are V band measurements. Glitch in phase around $53 \mathrm{GHz}$ is due to coaxial to waveguide adapter imperfection as it was optimized for $55 \mathrm{GHz}$ to $65 \mathrm{GHz}$.

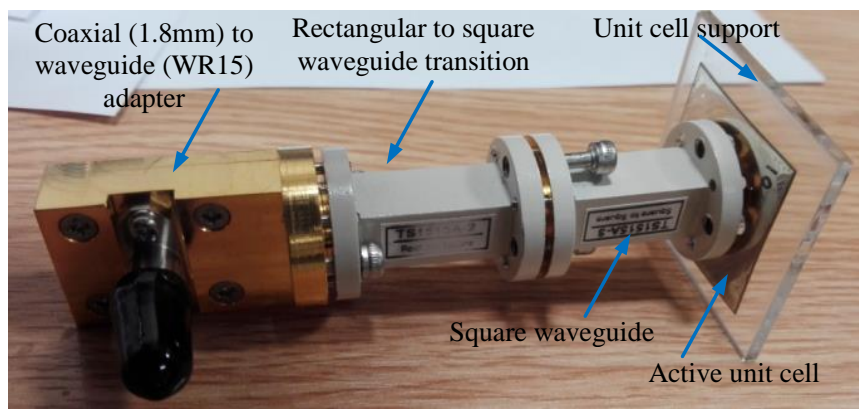

Fig. 8. Waveguide measurement setup for a multilayer active unit cell

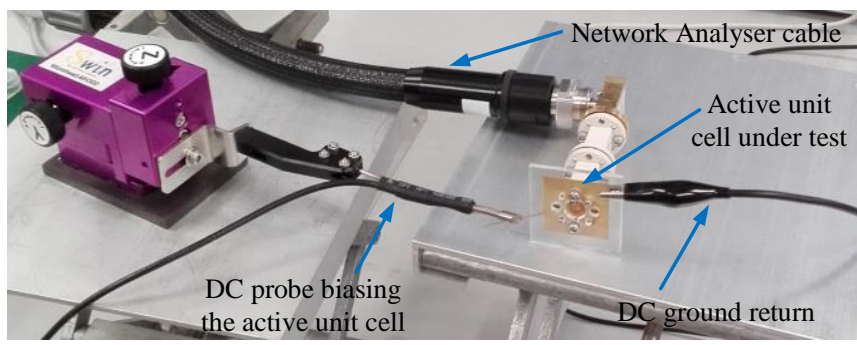

Fig. 9. Active unit cell under test, DC probe's needle is making contact with the DC biasing pad at rear of the active unit cell.

\begin{tabular}{|l|c|c|c|}
\hline Unit Cell Type & Fig.4 (a) & Fig.4 (b) & Fig.4 (c) \\
\hline Parameter & 59.23 & 59.35 & 59.04 \\
\hline Frequenant frequency (GHz) & 0 & 0.12 & -0.19 \\
\hline Phase deviation (Deg) & 0 & 6 & -10 \\
\hline
\end{tabular}

Table 1: Comparison of measured results for unit cell types shown in Fig. 4 (a)-(c). Unit cell measurement shown in Fig. 5 was taken as a reference case.

The waveguide structure used in passive and active unit cell measurements is shown in Fig. 8. While shifting from passive to active unit cell measurements, only the unit cell is replaced whereas rest of the structure remains same. There is no special need for any additional structure that requires customization. Fig. 9 displays the DC biasing mechanism for the active unit cell, where a DC probe with its needle makes an electrical contact with DC pad of the unit cell on its rear side. 


\section{Conclusion}

At mm-waves unit cells of a reflectarray become physically tiny and their characterization becomes significantly difficult. Additionally, the placement of unit cells inside the waveguide test setup need customized waveguide parts. The issue gains even more significance when it comes to the testing of active unit cells at mm-waves. This contribution provided two alternate solutions for unit cell characterization. The findings are equally valid for active as well as passive unit cells. Following the proposed structures reflectarray unit cell measurements are greatly simplified and placement/orientation issues of the unit cell in the waveguide gets resolved. DC biasing for active unit cells inside the waveguide has been made significantly simpler. Such findings would be particularly of interest for realization of low cost high gain reflectarray antenna solutions for next generation communication systems.

\section{Acknowledgements}

This research was jointly sponsored by Surrey Space Centre; University of Surrey and National Physical Laboratory under the project code SSC_NPL_KB4545 as a part of research on mm-wave reflectarray antennas. The work of T. H. Loh was supported by the 2013 - 2016 and 2016 - 2017 Quantum, Electromagnetics and Time program of the National Measurement Office, an Executive Agency of the U.K. Department for Business, Energy and Industrial Strategy, under Projects 116774 and 119575. Authors are highly indebted to Martin Salter and Nick Ridler from NPL, for their great support during the measurement campaign. Authors are thankful to Richard Clarke, Eric, and Graham Webb; from
University of Surrey Technical Support Unit (TSU), for their support during the fabrication of unit cells. Authors also want to extend their gratitude to Dr Peter Aaen and Haris Votsi from N3M laboratory of Advance Technology Institute (ATI) at University of Surrey for their continuous support during the measurements.

\section{References}

[1] K. K. Karnati, Y. Yusuf, S. Ebadi, and X. Gong, "Theoretical analysis on reflection properties of reflectarray unit cells using quality factors," Antennas and Propagation, IEEE Transactions on, vol. 61, pp. 201210, 2013.

[2] J. Perruisseau-Carrier, "Dual-polarized and polarization-flexible reflective cells with dynamic phase control," IEEE Transactions on Antennas and Propagation, vol. 58, pp. 1494-1502, 2010.

[3] J. Perruisseau-Carrier and P. Pardo, "Unit cells for dual-polarized and polarization-flexible reflectarrays with scanning capabilities," in Antennas and Propagation, 2009. EuCAP 2009. 3rd European Conference on, 2009, pp. 1218-1221.

[4] E. Carrasco, M. Barba, and J. A. Encinar, "X-band reflectarray antenna with switching-beam using PIN diodes and gathered elements," IEEE Transactions on Antennas and Propagation, vol. 60, pp. 5700-5708, 2012.

[5] D. Rodrigo, L. Jofre, and J. Perruisseau-Carrier, "Unit cell for frequency-tunable beamscanning reflectarrays," IEEE Transactions on Antennas and Propagation, vol. 61, pp. 5992-5999, 2013.

[6] H. Legay, Y. Cailloce, O. Vendier, G. Caille, J. Perruisseau-Carrier, M. Lathi, J. P. Polizzi, U. Oestermann, P. Pons, and N. Raveu, "Satellite antennas based on MEMS tunable reflectarrays," in Antennas and Propagation, 2007. EuCAP 2007. The Second European Conference on, 2007, pp. 1-6.

[7] H. A. Haus, "Waves and fields in optoelectronics," 1983.

[8] S. V. Hum and J. Perruisseau-Carrier, "Reconfigurable reflectarrays and array lenses for dynamic antenna beam control: A review," Antennas and Propagation, IEEE Transactions on, vol. 62, pp. 183-198, 2014.

[9] J. Huang, Reflectarray antenna: Wiley Online Library, 2008. 